\title{
Renin-angiotensin system research: from molecules to the whole body
}

\author{
Eriko Takimoto-Ohnishi ${ }^{1,2} \cdot$ Kazuo Murakami $^{1}$
}

Received: 8 December 2018 / Accepted: 17 April 2019 / Published online: 26 April 2019

(c) The Physiological Society of Japan and Springer Japan KK, part of Springer Nature 2019

\begin{abstract}
Hypertension is one of the most important risk factors and a leading cause of death from cardiovascular and cerebrovascular diseases. Based on numerous previous studies, hypertension is thought to be caused by the complex mutual interactions of genetic factors and environmental factors, such as excessive salt intake and stress. However, its detailed mechanisms are not yet clearly understood. The renin-angiotensin system (RAS) is a key hormonal system in the pathogenesis of hypertension. New knowledge is still accruing on this cascade, even after more than 120 years since the discovery of renin. To clarify the molecular mechanisms of RAS in vivo, we created transgenic mice with chronic hypertension. These mice carry the human genes encoding renin, a hypertensive enzyme, and its substrate angiotensinogen. Hypotensive mice homozygous for a targeted disruption of the angiotensinogen gene were also created. This review presents our 47-year history of RAS research.
\end{abstract}

Keywords Hypertension $\cdot$ Renin $\cdot$ Angiotensinogen $\cdot$ Renin-angiotensin system $\cdot$ Transgenic mice $\cdot$ Knockout mice

\section{Introduction}

Hypertension is the most prevalent clinical symptom, affecting 1.13 billion people worldwide [1]. The number of people with uncontrolled hypertension has risen over the years because of population growth and ageing. Complications of hypertension are thought to cause 9.4 million deaths each year, and without further action the World Health Organization (WHO) has predicted this number will increase [2, 3]. The renin-angiotensin system (RAS) is a regulatory cascade that plays major physiological roles in blood pressure regulation and electrolyte homeostasis (Fig. 1). Renin (EC 3.4.23.15), an aspartyl protease, catalyzes the specific cleavage of angiotensinogen to decapeptide angiotensin I (AI), the first and rate-limiting step in the RAS. The biologically

Eriko Takimoto-Ohnishi

onishi-e@ncchd.go.jp

$\triangle$ Kazuo Murakami murakami-k@msg.biglobe.ne.jp

1 Bio-Laboratory, Foundation for Advancement of International Science, 3-24-16 Kasuga, Tsukuba, Ibaraki 305-0821, Japan

2 Department of Maternal-Fetal Biology, National Center for Child Health and Development, 2-10-1 Okura, Setagaya-ku, Tokyo 157-8535, Japan inactive $\mathrm{AI}$ is then converted by angiotensin-converting enzyme (ACE) to the bioactive octapeptide, angiotensin II (AII). AII acting via its receptors induces a variety of physiological effects such as vasoconstriction, increasing cardiac output and promoting aldosterone synthesis. Dysregulation of the RAS plays a pivotal role in the pathogenesis of hypertension. Clinical evidence for the impact of RAS blocking agents suggested that renin-dependent mechanisms may be involved in more than $70 \%$ of patients with essential hypertension [4]. Most recently, a machine learning of big data on the treatment of hypertension has identified that ACE inhibitors and angiotensin receptor blockers were the most common treatment, used by $73 \%$ of the patients, as single drugs as well as in combinations with other antihypertensive medications [5]. Although it is beyond doubt that RAS blockers effectively lower blood pressure, the beneficial effects of that cannot be simply explained on the basis of changes in the circulating RAS. Recent evidence demonstrated that these blockers have other pleiotropic properties independent of their hypotensive effects, such as enhancement of cognition [6].

The complexity of the RAS has increased considerably in the past two decades [7]. This system was originally believed to be exclusively a circulating system, but because components of the RAS have been demonstrated in a variety of tissues, local RAS has been proposed as a paracrine function 


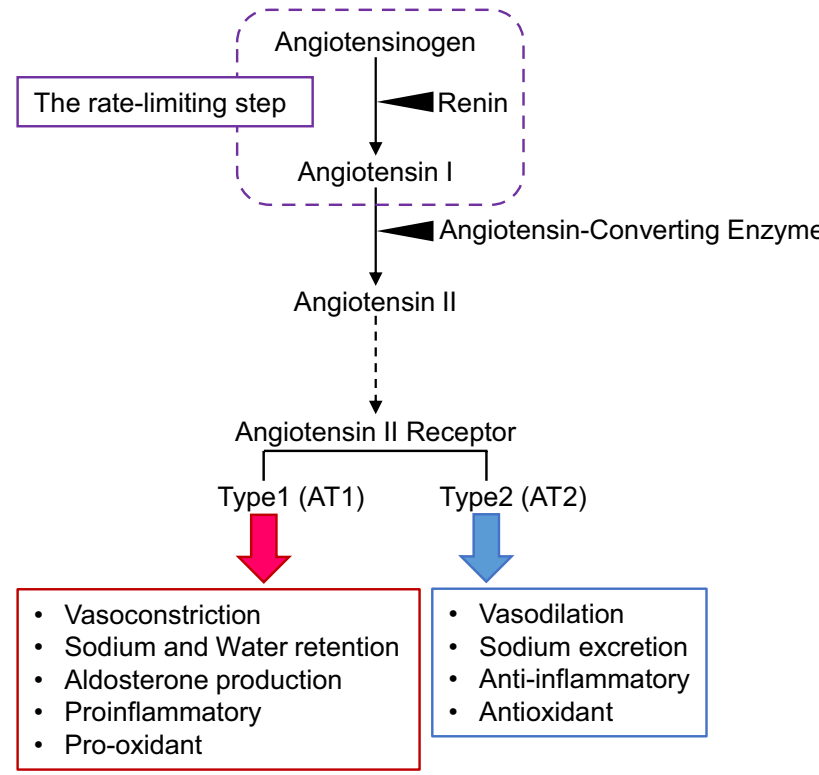

Fig. 1 RAS pathway and the role of its receptors. The first and ratelimiting step is a proteolysis of angiotensinogen by renin. Angiotensin I is further converted into angiotensin II, which mediates a variety of physiological effects through binding to its receptors

with physiological importance to the tissues in which it is expressed. In addition to vascular and renal actions, AII has direct effects at the cellular level impacting cell survival, differentiation and even inflammation. For more detailed information, the reader is referred to previous excellent reviews [6-8]. In this review, we present our long-standing research on the RAS at molecular, cellular, and whole-body levels.

\section{Decoy strategy}

Since approximately 1972, Murakami had expended great effort in isolating renal renin, which formed the core of renin research under the supervision of Professor Tadashi Inagami of Vanderbilt University, USA. However, the isolation of this protein was not an easy task and had been attempted by many other scientists in the past. Murakami also experienced difficulties and almost gave up. Fortunately, a new method for protein and enzyme isolation was being developed at that time. The principle was simple, and Murakami called this approach the "decoy strategy". Murakami discovered a "decoy substance", a ligand with a specific affinity for the target protein to be isolated (in this case, renin), and immobilized this ligand on an insoluble matrix. This affinity medium offered high selectivity for isolating renin (affinity chromatography).

For the first time, renin was successfully extracted from the kidney in its pure form using this affinity matrix. However, the basic structure of renin (i.e., its amino acid sequence) was impossible to determine due to a very small amount of the purified protein. This limitation was a substantial obstacle to our research, and we were completely defeated in our first attempt. Nevertheless, we now believe that this obstacle was actually a fortunate occurrence because the difficulty in isolating renin protein prompted Murakami to decide on the early introduction of new genetic engineering techniques. Using this breakthrough, biochemical research on renin entered a dramatic phase of expansion.

\section{Introduction of genetic engineering technology}

Five research groups worldwide, including ours, started projects to clone the mouse submandibular renin cDNA almost simultaneously. First, in 1982, a group at the Pasteur Institute obtained the full-length cDNA of mouse submandibular renin and published the complete nucleotide sequence and the entire predicted amino acid sequence.

The cloning of the human renin cDNA was much more challenging than the cloning of the mouse submandibular renin cDNA because normal amounts of human renal renin protein and mRNA are estimated to constitute less than approximately $1 / 100$ th the amount of mouse submandibular renin. Moreover, human renal renin mRNA was predicted to be as low as approximately $0.01-0.001 \%$ of the total mRNA. The lower the amount of the target mRNA content, the more difficult the cloning process becomes. This percentage was at the limits of the cDNA cloning technique at the time. However, we were able to obtain a sample that contained approximately 10 -fold more human renin mRNA than normal samples. A cDNA library was created from the extracted mRNAs. We were able to obtain the cooperation of the group led by Professor Shigetada Nakanishi of Kyoto University to synthesize the cDNA. We obtained the complete nucleotide sequence of the human renin cDNA and the complete human renin amino acid sequence. We then discovered the genomic sequences of human renin and its substrate, human angiotensinogen, as well as the promoter sites that control their expression [9-11]. One hundred years after its discovery, the basic structure (amino acid sequence) and genetic structure (complete nucleotide sequence) of human renin were finally published.

\section{Two lucky encounters}

Murakami's encounter with renin was actually completely by chance, and it occurred during his study as a visiting research fellow. Professor Stanley Cohen, who is famous for his epidermal growth factor (EGF) research, came into the laboratory and said excitedly "EGF is not only a growth factor for cells but also a factor that elevates blood pressure". Therefore, Murakami conducted studies to confirm whether EGF truly exerted these two effects. Six months later, he discovered that the substance that exerted the hypertensive 
effect was not EGF itself but a renin-like factor present in the mouse submaxillary gland.

"Heidelberg" was one more stroke of luck prerequisite for the success of human renin research. Murakami had spent 2 years of his time as a student in the dormitory of Kyoto University with a popular song called "Alt-Heidelberg". By coincidence, Murakami met Professor Shigetada Nakanishi for the first time 20 years after graduation in the town of Heidelberg in Germany. Without this chance meeting, his success in renin research would not have been possible. Furthermore, we received the Max Planck Research Award from The Alexander von Humboldt Foundation for our research findings following the start of the collaboration with Professor Detlev Ganten of the University of Heidelberg.

\section{Research on the whole body}

In vitro research on the molecules and cells identified to date has drastically improved as a result of new technological developments. However, molecular and cellular studies do not resolve the mechanism by which genes directly control blood pressure. Fortunately, these limitations were overcome by implementing an advanced method called genetic engineering and reproductive technologies. We visited Dr. Tatsuji Nomura (Head, Central Institute for Experimental Animals), as we wanted to introduce this revolutionary technology into our research. We explained our research plans and were able to start experiments with his kind assistance. We describe this technology next.

\section{Research methods}

\section{Creating transgenic mice}

Target DNA elements are injected into the pronucleus of fertilized ova obtained by natural breeding or by superovulation under a microscope. These injected and fertilized ova are then transplanted into the fallopian tubes of pseudopregnant mice. Following ontogenesis, DNA is extracted from the tail of the resulting progeny, and the introduction of the transgene is confirmed. Introduction of cloned genes into mice is a powerful approach to the study of gene regulation, because it permits functional studies of potential cisacting DNA elements involved in gene regulation in vivo in a normal developmental environment. In addition, transgenic animals provide an attractive biological system for analyzing the roles of the introduced gene product.

\section{Creating gene knockout mice}

Embryonic stem (ES) cells capable of differentiating into all cell types are subcultured in vitro. A target vector containing a desired mutation of a gene is inserted into ES cells by electroporation. After homologous recombination in the target locus, ES cells with the mutated gene are screened, and then the ES cells are injected into eight-cell embryos of recipient mice. The body of each chimeric mouse consists of cells derived from the altered ES cells and cells derived from the host embryo. If ES cells differentiate into germ cells in the chimeric mice, mice in which all the cells have the mutated gene can be obtained by crossing chimeric mice with wild-type mice. Gene targeting can be used to "knock out" individual component genes of the RAS. Gene targeting may provide revolutionary and exciting findings regarding the nature of the RAS in vivo.

\section{Transgenic mice carrying the human renin gene}

Our first challenge was to introduce the human renin gene into mice [11]. The promoter region required to regulate expression of the human renin gene was identified using cultured cells to ensure proper function of the human gene in transgenic mice. Based on this result, we produced a $15-\mathrm{kb}$ DNA fragment, with up to $3 \mathrm{~kb}$ of 5 '-flanking sequence. This DNA was then introduced into fertilized mice ova of the C57BL/6 J strain. The transgenes have been shown to be stably transmitted to progeny in accordance with the Mendelian laws of genetic inheritance. The human renin mRNA was expressed in the mouse kidney and its protein was also synthesized. As predicted, human renin was localized to the mouse juxtaglomerular cells. Moreover, the expression level was increased by 7.5 -fold in the mouse kidney compared with the human kidney. Despite the overexpression of human renin in the transgenic mice, their blood pressure was equal to that of the wild-type mice.

\section{Transgenic mice carrying the human angiotensinogen gene}

Our next challenge was to introduce the human angiotensinogen gene into mice [11]. We therefore identified the gene promoter region at the cellular level. The $1.3 \mathrm{~kb}$ of $5^{\prime}$-flanking region was designated as the promoter, and a $14-\mathrm{kb}$ DNA fragment containing the human promoter region was produced. This DNA was introduced into fertilized mice ova of the C57BL/6J strain to create mice carrying the human angiotensinogen gene. The transgenes have been shown to be stably transmitted to progeny, and approximately 100 copies of the exogenous gene were inserted into a single chromosomal locus. The human angiotensinogen gene was expressed at high levels in the liver of the transgenic mice. Nevertheless, the blood pressure of these transgenic mice was equal to that of the wild-type mice as well. 


\section{Characteristics and usefulness as a disease model}

\section{Tsukuba hypertensive mice}

Transgenic mice with either the human renin gene or the human angiotensinogen gene did not develop hypertension despite the normal tissue-specific expression of these transgenes. These results were caused by the strict speciesspecific reactivity between renin and angiotensinogen [12]. Therefore, in the next step, we crossed human renin transgenic mice and human angiotensinogen transgenic mice to create mice carrying both the human genes [13]. The dual human-gene strains were obtained through normal breeding methods, and we confirmed that expression of the human gene products was present in the plasma of these mice. The circulating levels of AI were increased by 3-4 fold compared to wild-type mice, confirming that the human enzyme-substrate reaction was actually occurring in mice. Furthermore, the increase in AI levels in the dual human-gene strains led to elevated levels of AII, which is the final physiologically active peptide, in the circulation. As a result of noninvasive measurement, the systolic blood pressure was $100 \mathrm{mmHg}$ on average in the wild-type and single human-gene strains, with no differences between strains. However, the systolic blood pressure was significantly elevated to an average of $130 \mathrm{mmHg}$ in the dual human-gene strains. The transgenic mice carrying both the human genes were thus named "Tsukuba hypertensive mice". The blood pressure of Tsukuba hypertensive mice decreased dramatically upon the administration of drugs that selectively inhibit human renin. On the other hand, a decrease in that was not observed in the wild-type and single human-gene strains upon the administration of the same drug. Thus, in the Tsukuba hypertensive mice, AI produced from the specific reaction between human renin and human angiotensinogen is converted to AII by endogenous ACE in the mice, subsequently causing hypertension.

The RAS is involved in the development of hypertension but is also reported to possibly play a central role in cardiac enlargement and the development of arteriosclerosis. Interestingly, many of our Tsukuba hypertensive mice developed cardiac enlargement before reaching 3 months of age, which became more apparent with aging. Mice frequently progressed to myocardial cell enlargement, resulting in vascular rupture of the cervical aortic arch in some cases. Furthermore, glomerulosclerosis occurred frequently after the age of 6 months [14]. The consumption of a high-fat and high-cholesterol diet also resulted in the prominent development of atherosclerosis in Tsukuba hypertensive mice [15]. Based on these findings, the Tsukuba hypertensive mouse strain is a very useful model for analyzing the physiological and pathophysiological role of the RAS not only in hypertension but also in complications of hypertension such as cardiovascular and renal dysfunction.

\section{Pregnancy-induced hypertension}

As described above, the two lines of single human-gene strains were normotensive despite the normal tissuespecific expression pattern of transgenes. In contrast, the progeny born by cross-mating the two lines, expressing the dual human-genes, exhibited a chronic hypertension [13]. Surprisingly, the cross-mating experiments brought us an unexpected discovery. We observed that transgenic females carrying the human angiotensinogen gene, which displayed normal blood pressure in the nonpregnant state, developed hypertension in late pregnancy, but only when they had been mated with transgenic males carrying the human renin gene. More than half of the hypertensive pregnant mice died of vascular rupture in the aortic arch or heart failure [16].

Preeclampsia is a clinical syndrome characterized by the acute onset of hypertension and damage to multiple organs in late pregnancy. Although preeclampsia has a marked influence on maternal and infant health, there are no effective treatments. Pregnancy induces extensive maternal adaptations in cardiovascular and renal physiology. Circulating levels of renin and angiotensin proteins markedly increase during pregnancy. Alterations of the RAS have long been suspected to contribute to pregnancy-associated hypertension. The presence of renin in the placenta indicates that there is local renin synthesis and suggests the possible involvement of renin in the regulation of maternal blood pressure and uteroplacental vascular resistance and blood flow. However, there is no direct in vivo evidence that renin is secreted from the placenta into the maternal circulation and contributes to the regulation of maternal blood pressure.

Based on these facts, we hypothesized that the combined action of placental renin and maternal angiotensinogen might be the cause of the onset of hypertension in our transgenic pregnant mice. To test this hypothesis, we measured plasma human renin concentrations. The human renin levels gradually increased throughout pregnancy as did the accumulation of human renin production in the placenta of females mated with the human renin transgenic males. The blood pressure of the human angiotensinogen transgenic females mated with the human renin transgenic males began to increase at 14 days of gestation, continued to rise until the day before delivery, and returned to the level seen in the nonpregnant state by 3 days after delivery. In contrast, control pregnant mice did not show any change in blood pressure throughout pregnancy. Compared to normotensive pregnant mice, hypertensive pregnant mice had elevated plasma levels of AII. In addition, treatment of the transgenic hypertensive females with an inhibitor specific for human renin reduced 
blood pressure. These results clearly demonstrate paternally derived human renin produced in the placenta is secreted into the maternal circulation, and the combined action of placental renin and maternal angiotensinogen could induce hypertension in the transgenic pregnant mice.

Histopathologic examination also revealed myocardial hypertrophy, and uniform enlargement of glomeruli associated with an increase in urinary protein excretion. The hypertensive pregnant mice exhibited placental ischemic changes and their fetuses displayed growth restriction in size and weight. This mouse is thought to be a useful model for analyzing the pathogenic mechanism of preeclampsia [17], as has been shown in a recent study [18].

Now, we do the opposite type of mating combination in order to generate Tsukuba hypertensive mice. It is worth noting that phenotypic defects of the mothers and fetuses are not developed in the opposite type of mating combination, despite the overexpression of human renin and human angiotensinogen detected in the fetal placenta of both types of crossbreeding. To address the discrepant phenotypes, we analyzed transgene localization in the labyrinth and its permeability to the maternal circulation [19].

In the mouse placenta, gas and nutrient exchange occurs in the labyrinth, which is composed of highly branched fetal vasculature and trophoblast-lined maternal blood spaces. Trophoblasts in humans and mice share common patterns of expression for genes that have been shown to be critical for either the development or function of the cells. Our detailed analysis revealed that human renin produced in trophoblast cells was secreted into the maternal circulation, whereas human angiotensinogen produced in trophoblasts and trophoblastic epithelium could not pass through the selective barrier, probably due to their distinct spatial and temporal expression in the labyrinth. Although further experiments are needed for a decisive conclusion, these results provide in vivo evidence that the cell-specific expression of renin and angiotensinogen in the feto-maternal interface impacts their differential roles in pregnancy.

\section{Tsukuba hypotensive mice}

Gene knockout mice were first reported by Capecchi in 1989 by homologous recombination in ES cells. Using the gene targeting technology, we disrupted angiotensinogen, the only substrate for renin [20]. Heterozygous mutant mice lacking the angiotensinogen gene in one allele were produced first, and were used to make homozygous mutant mice lacking the angiotensinogen gene in both alleles. The plasma levels of angiotensinogen were 58\% lower in the heterozygous mutants. On the other hand, the levels were below detection in the homozygous mutants. Furthermore, the plasma levels of AI were also below the detection limit in homozygous mutants, confirming that these mutant mice lacked the substrate for renin and that the RAS was dysfunctional in the whole body. There was no significant difference in blood pressure between heterozygous mutant and wild-type mice. However, homozygous mutant mice clearly had chronic hypotension, with $34 \mathrm{mmHg}$ lower systolic blood pressures than their wild-type littermates, and this strain was named the "Tsukuba hypotensive mouse". These results clearly demonstrated that no other system implicated in the control of hypertension can replace the RAS, and the RAS plays an important role in regulating normal blood pressure.

Surprisingly, we further discovered that the Tsukuba hypotensive mouse strain exhibited abnormalities in brain functions. First, these mice displayed a reduction of depressive-like behavior in the behavioral despair swim tests and spontaneous locomotor activity diminished compared with wild-type mice, suggesting that angiotensinogen in the central nervous system may regulate the depressant state in the brain [21]. Second, the Tsukuba hypotensive mice showed an impaired blood-brain barrier function [22]. Although these abnormalities were rescued by administration of neuroactive angiotensin peptides (AII or AIV), the restoration of blood-brain barrier function was not inhibited by AII type 1 and type 2 receptor (AT1 and AT2) antagonists. Based on these findings, astrocytes with angiotensins may be required for functional maintenance of the blood-brain barrier.

\section{Renin gene knockout mice}

To verify the possibility of the presence of other angiotensin-forming enzymes in vivo, we next performed deletion of the renin gene [23]. Homozygous mutant mice showed neither detectable levels of plasma renin activity nor plasma AI. The systolic blood pressure of them was decreased by $20-30 \mathrm{mmHg}$ compared with that of wild-type mice. Increased volumes of urine and water intake and morphological changes in the kidney were observed in these mice, and the results were very similar to those of the Tsukuba hypotensive mice. Surprisingly, however, abnormalities of blood-brain barrier function and the decreased density of hippocampal cells observed in the Tsukuba hypotensive mice [24] were not observed in the homozygous renin mutants, suggesting the possible existence of an angiotensinforming enzyme other than renin in the murine brain.

\section{All receptor gene or ACE gene knockout mice}

At least two types of AII receptors have been identified; furthermore, AT1 are divided into the 1a and 1b subtypes. Most of the previously known effects of AII are predicted to be mediated by AT1. In 1995, we created mice lacking the ATla gene, which is expressed in multiple organs, to verify this hypothesis and identify the roles of the $1 \mathrm{a}$ and $1 \mathrm{~b}$ subtypes [25]. The systolic blood pressure was decreased 
by $10 \mathrm{mmHg}$ in the heterozygous mutants and by $22 \mathrm{mmHg}$ in the homozygous mutants compared with wild-type mice. Thus, AT1a plays an important role in regulating blood pressure in the RAS. Similar results were reported by Ito et al. in the same year [26].

In contrast, Ichikawa et al. showed that the blood pressure of the $A T 1 b$ gene knockout mice was equivalent to that of the wild-type mice, and no morphological abnormalities in the kidney were observed [27]. They also created a doubleknockout mouse that lacked both the ATI $a$ and ATIb genes [28]. Similar degrees of decrease in blood pressure and morphological abnormalities in the kidneys were observed in these mice compared to angiotensinogen knockout mice. Therefore, the main physiological effects of AII are exerted via AT1. However, single deletion of ATla or ATIb did not result in morphological abnormalities in the kidneys, and thus, the authors concluded that AT1 a and 1b exhibit complementary activities. Furthermore, Inagami et al. succeeded in creating a knockout mouse that lacks the gene encoding AT2, which was created to elucidate the physiological role of this receptor [29]. Because AT2 is expressed at high levels in the fetal skin, it was believed to play an important role in fetal development or in associated processes. To our surprise, intravenous injections of AII exerted a drastic hypertensive effect on the AT2 gene knockout mice. These results show that AII activates AT1 and AT2, which have mutually counteracting hemodynamic effects. In addition, other groups succeeded in creating ACE knockout mice and demonstrated the role of this enzyme in systemic blood pressure, renal development and function, and male fertility [30, 31].

\section{Summary}

Our renin research, starting with biochemical isolation, has been substantially expanded by the introduction of molecular and developmental engineering technologies. Transgenic and gene targeting approaches continue to provide critical insights into the physiological and pathophysiological roles of the RAS in vivo. Among many components in the RAS, renin is the key enzyme, which shows exact species-specific reactivity between humans and mice (Table 1). The unique construction of chimeric RAS resulted in elevated blood pressure in the Tsukuba hypertensive mice and led us to discover a new function of placental human renin. Furthermore, it is interesting to note that unexpected phenotypes were observed in several tissues, including the brain and kidney of our mice models [32]. In recent years, the function of local RAS existing in virtually every organ of the body has been drawing attention [6-8]. Therefore, studies on the RAS-dependent hypertensive and hypotensive mice models will not only focus on hypertension but also further reveal numerous previously unknown functions beyond blood pressure regulation.
Table 1 RAS-dependent hypertensive and hypotensive mice models

\begin{tabular}{llll}
\hline Mouse strains & Human RAS & Mouse RAS & SBP \\
\hline Wild-type mice & $-/-$ & $R N / A G$ & Normal \\
$h R N$ mice & $R N /-$ & $R N / A G$ & Normal \\
$h A G$ mice & $-/ A G$ & $R N / A G$ & Normal \\
$h R N \&$ \& $h G$ mice $^{\mathrm{a}}$ & $R N / A G$ & $R N / A G$ & Increased \\
$\mathrm{PIH}^{\mathrm{c}}$ & Paternal $R N /$ maternal & $R N / A G$ & Increased \\
& $A G$ & & \\
Non-PIH $^{\mathrm{d}}$ & Maternal $R N /$ paternal & $R N / A G$ & Normal \\
& $A G$ & & \\
$m A G-K O$ mice & $-/-$ & $R N /-$ & Decreased \\
$m R N-$ KO mice & $-/-$ & $-/ A G$ & Decreased \\
\hline
\end{tabular}

$R N$ renin gene, $A G$ angiotensinogen gene, - absence, $h$ - human, $m$ mouse, $K O$ knockout, $S B P$ systolic blood pressure, increased or decreased, versus wild-type mice

${ }^{a}$ Tsukuba hypertensive mice

${ }^{\mathrm{b}}$ Tsukuba hypotensive mice

${ }^{\mathrm{c}}$ Pregnancy-induced hypertensive mice (i.e., hAG-females mated with hRN-males)

${ }^{\mathrm{d} N o n-h y p e r t e n s i v e ~ t r a n s g e n i c ~ p r e g n a n t ~ m i c e ~(i . e ., ~ h R N-f e m a l e s ~}$ mated with hAG-males)

Acknowledgments We especially thank Drs. Akiyoshi Fukamizu, Kenichi Yagami, Fumihiro Sugiyama, Keiji Tanimoto (Tsukuba University) and Junji Ohnishi (Tokyo Kasei University). We also sincerely thank the members of our laboratory and collaborators.

Funding Our work was supported by grants from the Ministry of Education, Science, Sports, and Technology of Japan, the Japan Society for the Promotion of Science, the Ministry of Health, Labour, and Welfare of Japan, Circulation Biosystems at the University of Tsukuba, the Chichibu Cement Co. Ltd., Iwaki Foundation, Naito Memorial Foundation, Ciba-Geigy Foundation, Fumi Yamamura Memorial Foundation for Female Natural Scientists, Uehara Memorial Foundation, Kanae Foundation of Research for New Medicine, Inamori Foundation, Asahi Glass Foundation, Naito Foundation, Mochida Memorial Foundation for Medical and Pharmaceutical Research, and Nissan Science Foundation.

\section{Compliance with ethical standards}

Conflict of interest E. Takimoto-Ohnishi declares that she has no conflict of interest. K. Murakami declares that he has no conflict of interest.

Ethical approval All applicable institutional guidelines for the care and use of animals were followed.

\section{References}

1. World Health Organization (2019) Global Health Observatory data. https://www.who.int/gho/ncd/risk_factors/blood_press ure_prevalence/en/ Accessed 7 April 2019

2. World Health Organization (2013) A global brief on hypertension: silent killer, global public health crisis. World Health Day 2013. http://www.who.int/iris/handle/10665/79059 
3. Kjeldsen S, Feldman RD, Lisheng L, Mourad JJ, Chiang CE, Zhang W, Wu Z, Li W, Williams B (2014) Updated national and international hypertension guidelines: a review of current recommendations. Drugs 74:2033-2051

4. Steven A, Atlas MD (2007) The renin-angiotensin aldosterone system: pathophysiological role and pharmacologic inhibition. J Manag Care Pharm 13:S9-S20

5. Koren G, Nordon G, Radinsky K, Shalev V (2018) Machine learning of big data in gaining insight into successful treatment of hypertension. Pharmacol Res Perspect. https://doi. org/10.1002/prp2.396

6. Jackson L, Eldahshan W, Fagan SC, Ergul A (2018) Within the brain: the renin angiotensin system. Int J Mol Sci. https://doi. org/10.3390/ijms19030876

7. Mirabito Colafella KM, Danser AHJ (2017) Recent advances in angiotensin research. Hypertension 69:994-999

8. Muñoz-Durango N, Fuentes CA, Castillo AE, González-Gómez LM, Vecchiola A, Fardella CE, Kalergis AM (2016) Role of the renin-angiotensin-aldosterone system beyond blood pressure regulation: molecular and cellular mechanisms involved in end-organ damage during arterial hypertension. Int J Mol Sci. https://doi.org/10.3390/ijms17070797

9. Miyazaki H, Fukamizu A, Hirose S, Hayashi T, Hori H, Ohkubo H, Nakanishi S, Murakami K (1984) Structure of the human renin gene. Proc Natl Acad Sci USA 81:5999-6003

10. Fukamizu A, Takahashi S, Seo MS, Tada M, Tanimoto K, Uehara S, Murakami K (1990) Structure and expression of the human angiotensinogen gene. Identification of a unique and highly active promoter. J Biol Chem 265:7576-7582

11. Tamura K, Umemura S, Fukamizu A, Ishii M, Murakami K (1995) Recent advances in the study of renin and angiotensinogen genes: from molecules to the whole body. Hypertens Res 18:7-18

12. Fukamizu A, Takimoto E, Sugimura K, Hatae T, Seo MS, Takahashi S, Sugiyama F, Kajiwara N, Yagami K, Murakami K (1993) Dependence of angiotensin production in transgenic mice carrying either the human renin or human angiotensinogen genes on species-specific kinetics of the renin-angiotensin system. Arzneimittelforschung 43:222-225

13. Fukamizu A, Sugimura K, Takimoto E, Sugiyama F, Seo MS, Takahashi S, Hatae T, Kajiwara N, Yagami K, Murakami K (1993) Chimeric renin-angiotensin system demonstrates sustained increase in blood pressure of transgenic mice carrying both human renin and human angiotensinogen genes. J Biol Chem 268:11617-11621

14. Shimokama T, Haraoka S, Horiguchi H, Sugiyama F, Murakami K, Watanabe T (1998) The Tsukuba hypertensive mouse (transgenic mouse carrying human genes for both renin and angiotensinogen) as a model of human malignant hypertension: development of lesions and morphometric analysis. Virchows Arch 432:169-175

15. Sugiyama F, Haraoka S, Watanabe T, Shiota N, Taniguchi K, Ueno Y, Tanimoto K, Murakami K, Fukamizu A, Yagami K (1997) Acceleration of atherosclerotic lesions in transgenic mice with hypertension by the activated renin-angiotensin system. Lab Invest 76:835-842

16. Takimoto E, Ishida J, Sugiyama F, Horiguchi H, Murakami K, Fukamizu A (1996) Hypertension induced in pregnant mice by placental renin and maternal angiotensinogen. Science 274:995-998

17. Ishida J, Matsuoka T, Saito-Fujita T, Inaba S, Kunita S, Sugiyama F, Yagami K, Fukamizu A (2011) Pregnancy-associated homeostasis and dysregulation: lessons from genetically modified animal models. J Biochem 150:5-14

18. Nezu M, Souma T, Yu L, Sekine H, Takahashi N, Wei AZ, Ito S, Fukamizu A, Zsengeller ZK, Nakamura T, Hozawa A, Karumanchi SA, Suzuki N, Yamamoto M (2017) Nrf2 inactivation enhances placental angiogenesis in a preeclampsia mouse model and improves maternal and fetal outcomes. Sci Signal. https://doi.org/10.1126/scisignal.aam5711

19. Takimoto-Ohnishi E, Saito T, Ishida J, Ohnishi J, Sugiyama F, Yagami K, Fukamizu A (2005) Differential roles of renin and angiotensinogen in the feto-maternal interface in the development of complications of pregnancy. Mol Endocrinol 19:1361-1372

20. Tanimoto K, Sugiyama F, Goto Y, Ishida J, Takimoto E, Yagami K, Fukamizu A, Murakami K (1994) Angiotensinogen-deficient mice with hypotension. J Biol Chem 269:31334-31337

21. Okuyama S, Sakagawa T, Sugiyama F, Fukamizu A, Murakami K (1999) Reduction of depressive-like behavior in mice lacking angiotensinogen. Neurosci Lett 261:167-170

22. Kakinuma Y, Hama H, Sugiyama F, Yagami K, Goto K, Murakami K, Fukamizu A (1998) Impaired blood-brain barrier function in angiotensinogen-deficient mice. Nat Med 4:1078-1080

23. Yanai K, Saito T, Kakinuma Y, Kon Y, Hirota K, TaniguchiYanai K, Nishijo N, Shigematsu Y, Horiguchi H, Kasuya Y, Sugiyama F, Ki Yagami, Murakami K, Fukamizu A (2000) Renin-dependent cardiovascular functions and renin-independent blood-brain barrier functions revealed by renin-deficient mice. J Biol Chem 275:5-8

24. Kakinuma Y, Hama H, Sugiyama F, Goto K, Murakami K, Fukamizu A (1997) Anti-apoptotic action of angiotensin fragments to neuronal cells from angiotensinogen knock-out mice. Neurosci Lett 232:167-170

25. Sugaya T, Nishimatsu S, Tanimoto K, Takimoto E, Yamagishi T, Imamura K, Goto S, Imaizumi K, Hisada Y, Otsuka A, Uchida H, Sugiura M, Fukuta K, Fukamizu A, Murakami K (1995) Angiotensin II type 1a receptor-deficient mice with hypotension and hyperreninemia. J Biol Chem 270:18719-18722

26. Ito M, Oliverio MI, Mannon PJ, Best CF, Maeda N, Smithies O, Coffman TM (1995) Regulation of blood pressure by the type 1A angiotensin II receptor gene. Proc Natl Acad Sci USA 92:3521-3525

27. Chen X, Li W, Yoshida H, Tsuchida S, Nishimura H, Takemoto F, Okubo S, Fogo A, Matsusaka T, Ichikawa I (1997) Targeting deletion of angiotensin type 1B receptor gene in the mouse. Am J Physiol 272:F299-F304

28. Tsuchida S, Matsusaka T, Chen X, Okubo S, Niimura F, Nishimura H, Fogo A, Utsunomiya H, Inagami T, Ichikawa I (1998) Murine double nullizygotes of the angiotensin type 1A and $1 \mathrm{~B}$ receptor genes duplicate severe abnormal phenotypes of angiotensinogen nullizygotes. J Clin Invest 101:755-760

29. Ichiki T, Labosky PA, Shiota C, Okuyama S, Imagawa Y, Fogo A, Niimura F, Ichikawa I, Hogan BL, Inagami T (1995) Effects on blood pressure and exploratory behaviour of mice lacking angiotensin II type-2 receptor. Nature 377:748-750

30. Esther CR Jr, Howard TE, Marino EM, Goddard JM, Capecchi MR, Bernstein KE (1996) Mice lacking angiotensin-converting enzyme have low blood pressure, renal pathology, and reduced male fertility. Lab Invest 74:953-965

31. Krege JH, John SW, Langenbach LL, Hodgin JB, Hagaman JR, Bachman ES, Jennette JC, O'Brien DA, Smithies O (1995) Male-female differences in fertility and blood pressure in ACEdeficient mice. Nature 375:146-148

32. Murakami K, Fukamizu A (1999) Transgenic and knockout models in renin-angiotensin system. Immunopharmacology 44:1-7

Publisher's Note Springer Nature remains neutral with regard to jurisdictional claims in published maps and institutional affiliations. 